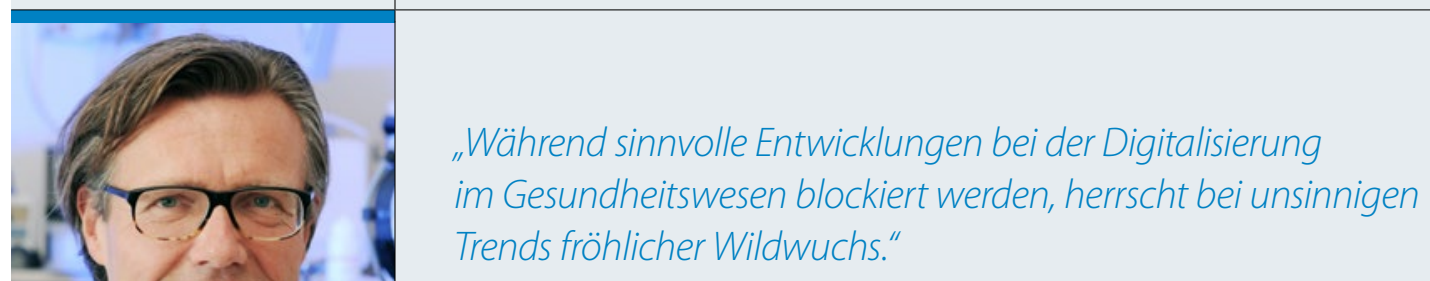

Prof. Dr. med Gerhard Grevers

Chefredaktion

\title{
Sinn und Unsinn der Digitalisierung
}

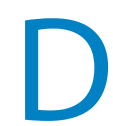
igitalisierung wird groß geschrieben in der Arbeitswelt, und das nun schon seit einiger Zeit. Selbst in bundesdeutschen Amtsstuben, die ja nicht wirklich als innovationsaffin gelten, ist der Bürger 2.0 mittlerweile zumindest ansatzweise angekommen. Eine Ausnahme bildet, wie so oft, das Gesundheitswesen, von bestimmten Kreisen hochgepriesen und doch überteuert, überreguliert und in weiten Bereichen insuffizient. Nach dem Rohrkrepierer Gesundheitskarte wird nun am E-HealthGesetz herumgedoktert, Leitragende sind, wie üblich, neben den Ärzten, die seit Jahrzehnten mit immer weiter zunehmendem bürokratischem Unsinn belastet werden, vor allem die Patienten. Letztere zahlen Rekordbeiträge an die Versicherungen, der Service in der Kundenbetreuung ist dafür lausig. Patienten beschweren sich über unbeantwortete EMails, Hotlines entpuppen sich als Synonym für endlose Warteschleifen, zurückgerufen wird in der Regel auch nicht. Zahlreiche Unzulänglichkeiten im Gesundheitswesen ließen sich mittels Digitalisierung beseitigen; in vielen, auch europäischen Ländern, ist man da schon viel weiter. Deutschland droht auch auf diesem Sektor wieder einmal den Anschluss zu verpassen. Gründe dafür gibt es genug; neben der Unbeweglichkeit der Akteure (Kassen, Kliniken, Ärzteschaft), deren Funktionäre sich $\mathrm{ja}$ in allen Bereichen des Gesundheitswesens in fast sado-masochistischer Manier permanent gegenseitig blockieren, wird gerne auch die "Datenschutz“Karte gezückt, ein Trumpf, mit dem sich in diesem Land praktisch jedes Projekt problemlos aushebeln lässt. Wohlgemerkt, Datenschutz ist wichtig und legitim, es gilt aber abzuwägen, wie lange man bereit ist, dafür wichtige Fortschritte zu verhindern.

Möglichkeiten, das System effektiver und patientenorientierter zu gestalten, gibt es viele. Durch die Digitalisierung existieren mittlerweile zahlreiche Ansätze, um Schwachstellen wie Bürokratisierung, Kundenservice etc. im deutschen Gesundheitssystem zu optimieren. In anderen Branchen geht das schließlich auch. Nur, dazu müsste Wettbewerb möglich sein, und die gesetzlichen Krankenversicherungen sind als Körperschaft des öffentlichen Rechts leider vor externen Wettbewerbern geschützt.

Regulierwahn auf der einen, Wildwuchs auf der anderen Seite. Während sinnvolle Entwicklungen bei der Digitalisierung im Gesundheitswesen konsequent blockiert werden, verbreiten sich Gesundheits-Apps völlig unkontrolliert. Mittlerweile soll es Angaben verschiedener Statistiken zufolge, so die Süddeutsche Zeitung, weltweit nahezu $380.000 \mathrm{Ge}-$ sundheits-Apps geben, Tendenz steigend. Allein in Deutschland sind zurzeit mehr als 100.000 Medizin-, Gesundheits- und Fitness-Apps verfügbar, davon 41.000 mit dem „engeren Thema“ Medizin. Kontrolle, was da so alles verbreitet wird? Fehlanzeige! Ob die Apps alle sinnvoll sind oder die eine oder andere nicht eher krank als gesund macht, interessierte bisher auch niemandem im ansonsten absurd überregulierten deutschen Gesundheitswesen. Zumindest das Bundesgesundheitsministerium (BGM) ist jetzt aufgewacht. Ergebnisse der bisher ersten vom BGM geförderten Studie wurden kürzlich von Forschern der Medizinischen Hochschule Hannover veröffentlicht; erwartungsgemäß heißt es dort, dass „qualitativ hochwertige Gesundheits-Apps eher die Ausnahme als die Regel" darstellen. „Die Studie ist eine wichtige Grundlage für den Fachdialog mit Experten und Verantwortlichen im Gesundheitswesen, in den wir nun eintreten wollen", schlussfolgert der zuständige Minister laut FAZ. ,Eintreten in den Dialog", da ist sie wieder, die Dynamik in der deutschen Politik!

Zeit wird's Freunde; sonst kann der unbedarfte „User“ solcher Apps leicht zum „Loser" werden, wenn's schlecht läuft ..., aber dann gibt's ja immer noch die „Hotline“ zur Krankenkasse, wenn man da nicht vorher in der Warteschleife verendet ...

In diesem Sinne wünsche ich Ihnen einen entspannten und erholsamen Sommer!

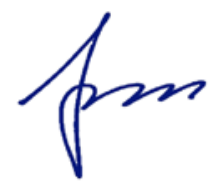

